\title{
Berberine improves hepatic insulin resistance by activating the SIRT1/Opa1 pathway: An in vitro and in vivo study
}

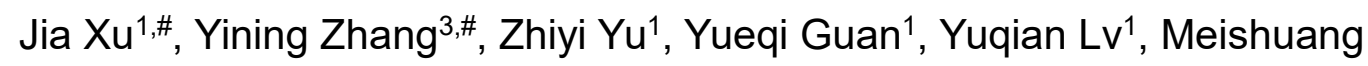
Zhang $^{1}$, Ming Zhang ${ }^{1}$, Li Chen ${ }^{1}$, Xiaoyan IV ${ }^{2, *}$, Fengying Guan ${ }^{1, *}$

1Department of Pharmacology, School of Basic Medical Sciences, Jilin University, Changchun 130021, China

2Department of Clinical Laboratory, the Second Clinical Hospital Affiliated to Jilin University, Changchun 130041, China

${ }^{3}$ Department of Pediatric Endocrinology, The First Clinical Hospital Affiliated to Jilin University, Changchun 130021, China

Running Title: Berberine improves hepatic insulin resistance via SIRT1/Opa1

\#Equal contribution

${ }^{*}$ Correspondence should be addressed to:

Fengying Guan

Tel: +86-431-85619799

E-mail: guanfy@jlu.edu.cn

Xiaoyan Lv

Tel: +86-431-81136607

E-mail: williams1980@jlu.edu.cn 


\section{Abstract}

Aim: This study explored whether abnormality in the inner mitochondrial membrane fusion protein optic atrophy 1 (Opa1) causes hepatic insulin resistance and whether berberine (BBR) can prevent hepatic insulin resistance through the SIRT1/Opa1 pathway. Method: High-fat diet (HFD)-fed mice and db/db mice were used as animal models to study hepatic insulin resistance in vivo. Insulin resistance, morphological changes, and mitochondrial injury of the liver were examined to explore the effects of BBR. SIRT1/Opa1 protein expressions were determined to confirm whether the signalling pathway was damaged in the model animals and involved in BBR treatment. A palmitate (PA)-induced hepatocyte insulin resistance model was established in HepG2 cells in vitro. Opa1 silencing and SIRT1 overexpression were induced to verify whether Opa1 abnormality causes hepatocyte insulin resistance and whether SIRT1 could improve this dysfunction. BBR treatment and SIRT1 silencing were employed to prove that BBR can prevent hepatic insulin resistance by activating the SIRT1/Opa1 pathway. Results: We found that Opa1 deficiency caused imbalance in mitochondrial fusion/fission and impaired insulin signalling in the HepG2 cells. SIRT1 and BBR overexpression ameliorated PA-induced insulin resistance, increased Opa1, and improved mitochondrial function. SIRT1 silencing could partly reverse the effects of BBR in the HepG2 cells. SIRT1 and Opa1 were downregulated in the animal models. BBR attenuated hepatic insulin resistance and enhanced SIRT1/Opa1 signalling in the the $\mathrm{db} / \mathrm{db}$ mice. Conclusion: Opal silencing-mediated mitochondrial fusion/fission imbalance could lead to hepatocyte insulin resistance. BBR may 
improve hepatic insulin resistance by regulating the SIRT1/Opa1 pathway, and thus, it may be used to treat type 2 diabetes.

Keywords: Berberine, Hepatic insulin resistance, SIRT1, Opa1, mitochondrial fusion/fission

\section{Introduction}

Mitochondria provide ATP to the cells [1] and play a key role in cellular energy metabolism. Mitochondrial dysfunction results in various metabolic diseases, including insulin resistance (IR) and type 2 diabetes [2] and is characterised by damaged substrate oxidation and impaired mitochondria accumulation, which are related to an imbalance in mitochondrial fusion/fission $[1,3]$. A few proteins of the GTPase family are involved in mammalian mitochondrial fusion/fission.

Outer mitochondrial membrane (OMM) fusion proteins include mitofusin (Mfn) 1 and 2, whereas optic atrophy 1 (Opa1) is an inner mitochondrial membrane (IMM) fusion protein. Dynamin-related protein 1 (Drp1) and fission protein 1 (Fis1) are responsible for mitochondrial fission [4]. The role of Mfn1, Mfn2, and Drp1 in hepatic IR has been investigated in studies. Sameer et al [5] reported that Mfn1 deficiency in the liver protects against diet-induced IR. David et al[6] observed that liver-specific Mfn2 $\mathrm{KO}$ mice had impaired glucose tolerance, enhanced hepatic gluconeogenesis, and impaired insulin sensitivity. Wang et al[7] observed that liver-specific Drp1 KO mice showed decreased fat mass and resistance to high-fat diet (HFD)-induced obesity. However, the relationship between Opal and hepatic IR as well as the specific molecular mechanisms of signal transduction have not been fully 
elucidated.

Sirtuin 1 (silent information regulator-1, SIRT1) regulates multiple biological processes, such as glucose-lipid metabolism and mitochondrial biogenesis[8]. SIRT1 can modulate hepatic glucose metabolism by interacting with PGC-1 $\alpha$ and directly increase insulin sensitivity in the liver under different pathological conditions[9]. Jung et al reported[10] that an increase in the expression of SIRT1 could ameliorate palmitate (PA)-induced hepatocyte IR. SIRT1 plays a key role in mitochondrial biogenesis through PGC-1 $\alpha$ acetylation/deacetylation[11]. Recent studies have proposed that SIRT1 regulates the transcription of Drp1 through PGC-1 $\alpha$ in diabetic hearts[12]. However, the role of SIRT1 in Opal regulation is unknown.

Berberine (BBR) is the main active ingredient of Coptis Root that can accelerate glucose transport, increase glycogen synthesis, regulate glucose metabolism, and improve hepatic IR[13]. Many studies have focused on exploring whether BBR can improve the mitochondrial function. Qin et al[14] revealed that BBR could protect glomerular podocytes by inhibiting Drp1-mediated mitochondrial fission and dysfunction. Qin et al[15] suggested that BBR regulates the excessive production of mitochondrial ROS and mitochondrial dysfunction by activating PGC- $1 \alpha$ and thus prevents diabetic nephropathy.

Therefore, we investigated the relationship between Opal and hepatic IR in the absence of Opa1 activation and examined whether SIRT1 regulates Opa1 to improve PA-induced hepatocyte IR with SIRT1 activation and Opa1 silencing in HepG2 cells. Finally, we explored whether BBR can improve hepatic IR by activating the 
SIRT1/Opa1 pathway through SIRT1 silencing.

\section{Materials and Methods}

\section{Animal Care}

Three-week-old male C57BL/6J mice (HFK Bioscience, Beijing) were raised in a standard temperature and humidity-controlled environment with a 12:12-h light-dark cycle for a week before experiments. The mice had access to nesting material and were provided ad libitum access to water and commercial low-fat diet (LFD, 10\% fat) or HFD, 60\% fat) (Research Diets, America) for 10 weeks.

Seven-week-old male $\mathrm{db} / \mathrm{db}$ mice and C57 mice were purchased from Model Animal Research Center of Nanjing University (Nanjing, China) and adaptively raised for a week in the same environment. These $\mathrm{db} / \mathrm{db}$ mice were fed a regular diet for 4 weeks. In the CON group, the C57 mice were given NS (10 mL/kg, b.w.). The $\mathrm{db} / \mathrm{db}$ mice were randomly divided into two groups. In the model group ( $\mathrm{db} / \mathrm{db})$ and BBR group, diabetic mice were given NS (10 ml/kg, b.w.) and BBR (160 mg/kg, b.w.), respectively. Tests were conducted to measure the body weight, fasting blood glucose (FBG), low-density lipoprotein cholesterol (LDL), triglycerides (TG), total cholesterol (T-CHO), fasting blood insulin, and glucose-stimulated insulin secretion (GSIS) of the mice. Additionally, the oral glucose tolerance test (OGTT) and insulin tolerance test (ITT) were conducted.

\section{Determination of OGTT, ITT, and GSIS}

After allowing the C57BL/6J mice to feed on LFD and HFD for 10 weeks and the $\mathrm{db} / \mathrm{db}$ mice to feed on a regular diet for 4 weeks, the mice were starved for $12 \mathrm{~h}$ 
before OGTT. Approximately $10 \mu \mathrm{L}$ of blood was drawn from the mice tail tip and OGTT was performed using test strips (Roche, America) and a glucometer. After providing oral feeding of $40 \%$ glucose $(2 \mathrm{~g} / \mathrm{kg}$, b.w., Xilong Scientific Company, China) to the mice, their blood glucose was tested at $0 \mathrm{~min}, 30 \mathrm{~min}, 60 \mathrm{~min}, 90 \mathrm{~min}$, and $120 \mathrm{~min}$. For ITT, the $\mathrm{db} / \mathrm{db}$ mice were starved for $2 \mathrm{~h}$ and then were administered insulin (0.5 IU/kg b.w., i.p, Novolin R, Novo Nordisk A/S, Denmark). Blood glucose was measured at $0 \mathrm{~min}, 15 \mathrm{~min}, 30 \mathrm{~min}, 60 \mathrm{~min}$, and $120 \mathrm{~min}$. For the GSIS test, blood was drawn from the iliac vein of the C57BL/6 $\mathrm{J}$ mice at 0 min and 30 min and insulin was measured using Mouse Ultrasensitive Insulin ELISA, according to manufacturer's instructions (No: 80-INSMSU-E01, ALPCO, America).

\section{HE Staining}

After anaesthetising the mice with $10 \%$ chloral hydrate, the liver tissue was immediately removed at $4{ }^{\circ} \mathrm{C}$, washed using ice-cold PBS solution, and then fixed in $10 \%$ formaldehyde solution for a day. The tissue was dehydrated with conventional gradient alcohol and embedded in paraffin. The sections were 5- $\mu \mathrm{m}$ thick. After dewaxing and hydration, haematoxylin dyeing, dehydration, and neutral gum sealing, a good field of view was selected to observe and capture photographs using an Olympus BX53 fluorescence microscope (Tokyo, Japan).

\section{Electron Microscopy}

Liver samples obtained from the $\mathrm{db} / \mathrm{db}$ mice were minced and placed in a fixative solution comprising $2.5 \%$ glutaraldehyde and $2 \%$ paraformaldehyde in $0.1 \mathrm{M}$ cacodylate buffer ( $\mathrm{pH} 7.4$ ) for $2 \mathrm{~h}$ at $4^{\circ} \mathrm{C}$. These samples were postfixed in $1 \%$ 
osmium tetraoxide buffer for $1.5 \mathrm{~h}$. The tissue samples were then dehydrated using alcohol and embedded in Epon. Sections of 80-nm thickness were prepared and photographed using the X-650 electron microscope (Hitachi, Japan). The mitochondria in hepatic cells were observed by Images.

\section{Cell Culture and Transfection}

HepG2 cells were cultured in DMEM (4.5 g/L glucose, gibco, America) supplemented with 10\% FBS (Clark, Australia). For immunofluorescence assays, the HepG2 cells were fixed using 4\% paraformaldehyde, and then, mitochondria were visualised by overexpressing mitochondrially targeted Mito-DsRed protein (Addgene, America). For the Opal knock-down experiment, the Opal siRNA sequence (GATCATCTGCCACGGGTTGTT) was purchased from Shanghai GenePharma (China). SIRT1 siRNA sequence (ACUUUGCUGUAACCCUGUATT) and overexpression plasmid were also purchased from Shanghai GenePharma (China).

\section{Detection of HepG2 Cell Viability using MTT Assay}

HepG2 cells were adjusted to a density of $5 \times 10^{3}$ cells $/ \mathrm{mL}$ and cultured in a 96-well microplate. After treatment with different concentrations of PA and BBR for $24 \mathrm{~h}, 20 \mu \mathrm{L}$ of MTT solution was added to each well and the cells were incubated for $3 \mathrm{~h}$. Then, $200 \mu \mathrm{L}$ DMSO solution was added. The absorbance was measured at 562 nm by using Thermo Scientific Microplate Reader (Multiskan Spectrum).

\section{Glucose Consumption}

Cell suspensions of the HepG2 cells in the logarithmic growth phase were prepared. A density of $10^{5}$ cells $/ \mathrm{mL}$ was adjusted, and $500 \mu \mathrm{L}$ of the suspension was 
seeded in each well of a 24 -well plate. When IR was induced in the HepG2 cells by exposure to $0.3 \mathrm{mM} \mathrm{PA}$ for $24 \mathrm{~h}$, the cells were transfected with SIRT1 overexpression plasmid (oe-SIRT1), Opa1 siRNA(si-Opa1), or SIRT1 siRNA(si-SIRT1) for $24 \mathrm{~h}$. The cells were divided into the following groups: normal $(\mathrm{CON})$, model (PA), PA + oe-SIRT1 or PA + BBR, and PA + oe-SIRT1 + si-Opa1 or $\mathrm{PA}+\mathrm{BBR}+$ si-SIRT1. According to the manufacturer's instructions, the glucose content of the supernatant in triplicate wells was measured using a glucose assay kit (glucose oxidase method, Shanghai Robio, China). Glucose consumption (mmol/L) was calculated as the difference in glucose concentration between the blank and test groups $($ glucose consumption $=$ glucose concentration of blank wells - glucose concentration of wells with cells)[16].

\section{Western Blot Analysis}

Equal amounts of proteins were separated on SDS-PAGE under reducing conditions and then transferred to PVDF membranes (Merck Millipore). After blocking with $5 \%$ skimmed milk in TBS supplemented with $0.1 \%$ Tween-20, the PVDF membranes were incubated with the following antibodies: mouse anti-SIRT1 (1:1000, Abcam), mouse anti-Drp1 (1:1000, Abcam), mouse anti-Opa1 (1:1000, Proteintech), rabbit anti-Mfn1 (1:1000, Abcam), rabbit anti-Mfn2 (1:1000, Abcam), mouse anti-NDUFA9 (1:1000, Proteintech), mouse anti-ATP5A1 (1:1000, Proteintech), rabbit anti-AKT (1:500, Santa), rabbit anti-pAKT (1:500, Santa), and rabbit anti-GAPDH $(1 ; 2000$, Proteintech). The signal was visualised using the corresponding horseradish peroxidase-conjugated secondary antibodies (1:5000, 
Proteintech) and enhanced chemiluminescence-western blotting detection reagent (Bioscience Biotech, Beijing). Bands were quantified through densitometry by using Quantity One software.

\section{Real-time Quantitative PCR}

Total RNA of liver tissues and that of HepG2 cells was extracted using Trizol reagent (Invitrogen, Shanghai, China), and the isolated RNA samples were subjected to reverse transcription by using superscript ${ }^{\circledR}$ III first-strand synthesis system (Invitrogen, Shanghai, China). Real-time PCR was performed using the MX 3000P Real-Time PCR instrument with SYBR Green Mix and the following primer sequences. Data were normalised relative to those for GAPDH or $\beta$-actin expression by using the $2^{-\Delta \Delta \mathrm{Ct}}$ method.

\begin{tabular}{|l|l|}
\hline mouse $\beta$-actin forward & GCTGAGAGGGAAATCGTGCGT \\
\hline mouse $\beta$-actin reverse & ACCGCTCGTTGCCAATAGTGA \\
\hline mouse PEPCK forward & AAAGCAAGACAGTCATCATCACCCA \\
\hline mouse PEPCK reverse & TCTCAAAGTCCTCTTCCGACATCC \\
\hline mouse G6pase forward & TTGCCAGGAAGAGAAAGAAGGAT \\
\hline mouse G6pase reverse & AACACAGACACAACTGAAGCCG \\
\hline homo GAPDH forward & CCATGGAGAAGGCTGGG \\
\hline homo GAPDH reverse & CAAAGTTGTCATGGATGACC \\
\hline homo PEPCK forward & AGCCTCGACAGCCTGCCCACGG \\
\hline homo PEPCK reverse & CCAGTTGACCAAAGGCTTTT \\
\hline homo G6pase forward & ACATCCGGGGCATCTACAATG \\
\hline
\end{tabular}




\section{Determination of the Cellular ATP Level}

The ATP level was measured using a firefly luciferase-based ATP assay kit (Beyotime, China), according to the manufacturer's instructions. The HepG2 cells were cultured and prepared, as described in the section 'glucose consumption'. The cells were rinsed using PBS solution, collected using ATP lysing agent, and then centrifuged for $5 \mathrm{~min}$ at $12,000 \mathrm{~g}$ at $4^{\circ} \mathrm{C}$. The supernatant was collected. In a $1.5-\mathrm{mL}$ tube, $100 \mu \mathrm{L}$ of the supernatant was mixed with $100 \mu \mathrm{L}$ of the ATP detection solution. Luminance (RLU) was immediately measured using an H1 synergy microplate reader. Standard curves for the quantification were also generated using the ATP standard, and the protein concentration of each treatment group was determined using the BCA protein assay kit (Thermo Scientific, USA). Total ATP levels were expressed as NRLU (nmol/mg protein).

\section{Assessment of Mitochondrial Membrane Potential}

Mitochondrial membrane potential assay kit (JC-1) was used to measure the mitochondrial membrane potential (MMP) of the HepG2 cells, according to the manufacturer's instructions (C2006, Beyotime Biotechnology, China). The cells were washed twice with PBS solution and then incubated with $\mathrm{JC}-1$ at $37^{\circ} \mathrm{C}$ for $30 \mathrm{~min}$. After incubation, the cells were washed again. OLYMPUS IX71 fluorescence microscope was used to observe the difference between green and red 
fluorescence. Red and green fluorescence represented high and low MMP, respectively.

\section{Statistical Analyses}

Statistical analyses were performed using the GraphPad Prism5 software. Differences between the two groups were analysed using the Student t-test (two-tailed, $\mathrm{P}<0.05$ was considered significant). Differences between multiple groups were analysed using the one-way analysis of variance followed by Dunnett's post hoc test $(\mathrm{P}<0.05$ was considered significant $)$. The data are expressed as means \pm SEM.

\section{Results}

Downregulation of SIRT1 and Opa1 was related to the imbalance of mitochondrial dynamics and hepatic IR

HFD-induced obese mice were used to examine the relationship between SIRT1/Opa1 signalling and hepatic IR. A significant increase was observed in the weight and FBG of the HFD-fed mice 10 weeks after their feeding on LFD and HFD (Fig. 1A and 1B). Moreover, the HFD-fed mice exhibited impaired glucose tolerance and hyperinsulinemia (Fig. 1C and 1D). Increased expressions of gluconeogenic enzymes [phosphoenolpyruvate carboxykinase (PEPCK) and glucose 6-phosphatase (G6pase)] were observed (Fig. 1E). The results of HE staining of the liver revealed that the cells in the HFD-fed mice were highly swollen, cellular matrix was highly porous and showed vacuole-like changes, hepatocytes had fatty vacuoles of various 
sizes and clear boundaries, and hepatocyte cords were disorderly arranged, which mainly manifested as cell oedema and hepatic steatosis (Fig. 1F). These results suggested that the mice had developed hepatic IR 10 weeks after feeding on HFD.

We observed that in the HFD-fed mice, the expression of the mitochondrial fusion proteins Opal and Mfn1 decreased, whereas that of the fission protein Drp1 increased (Fig. 2C, 2D and 2E), suggesting an imbalance of mitochondrial dynamics. The expression of SIRT1 decreased in the HFD-fed mice (Fig. 2B). Thus, we speculated that the downregulation of SIRT1 and Opa1 is related to the imbalance of mitochondrial dynamics and hepatic IR.

\section{Opa1 deficiency changed mitochondrial fusion/fission and impaired insulin signalling in the HepG2 cells}

To investigate the role of Opal in hepatic IR, we transfected HepG2 cells with Opa1-siRNA, and the transfection efficiency was approximately 55\% (Fig. 3A). First, we analysed whether Opal deficiency caused mitochondrial dysfunction. The expression level of OMM fusion proteins, Mfn1 and 2, remained unchanged, whereas that of fission protein, Drp1, increased (Fig. 3A). The expression level of NDUFA9 (mitochondrial complex I) decreased, whereas that of ATP5A (mitochondrial complex V) remained unchanged (Fig. 3A). The ATP concentration and MMP declined (Fig. 3B and 3C) upon Opal depletion.

After transfecting with Opa1-siRNA for $48 \mathrm{~h}$, two groups of cells were incubated with or without insulin $(100 \mathrm{nM})$ for $10 \mathrm{~min}$. IRS-2/PI3K/AKT is an important insulin signal pathway in the liver[17], whereas AKT phosphorylation is a marker for insulin 
sensitivity. Control cells displayed a marked increase in AKT phosphorylation in response to insulin, and this effect was found to be reduced in the Opa1-deficient cells (Fig. 3E). Moreover, the expression of PEPCK and G6pase in the Opa1-silenced HepG2 cells increased (Fig. 3D).

These results suggested that Opal deficiency increases mitochondrial fission, decreases mitochondrial ATP concentrations and membrane potential, and impairs insulin signalling and gluconeogenesis in HepG2 cells.

SIRT1 reversed hepatocyte IR and improved mitochondrial function in the HepG2 cells through Opa1-mediated mitochondrial fusion

SIRT1 has been reported to play a key role in IR and mitochondrial biosynthesis. Based on this information, we analysed the relationship between SIRT1 and Opa1. First, we screened the concentration of PA and established $0.3 \mathrm{mM}$ as the dose for the IR model (Fig. 4A and 4B). Based on the IR model, we transfected the SIRT1 overexpression plasmid and Opa1-siRNA and observed that SIRT1 activated Opa1 in the IR model (Fig. 4C and 4D). SIRT1 activated the pAKT/AKT expression, and this activation state could be reversed after transfection with Opal-siRNA (Fig. 4E). SIRT1 overexpression also increased glucose consumption in the IR model, and the state was also reversed after transfection with Opa1-siRNA. These results suggested that SIRT1 improves insulin signalling, hepatic glucose uptake, and gluconeogenesis in PA-induced IR, and this effect is achieved at least in part by Opal activation.

We investigated whether SIRT1 is linked to mitochondrial function during IR. SIRT1 overexpression increased ATP concentration and MMP (Fig. 4G and Fig. 5B), 
whereas Opa1 deletion partly blocked these effects. We investigated whether SIRT1 overexpression inhibits PA-induced mitochondrial fragmentation in the HepG2 cells by visualising mitochondrial morphology with Mito-DsRed protein. As shown in Fig. 5A, mitochondria of the control cells mainly appeared as elongated tubules, with highly interconnecting networks. After stimulation with PA for $24 \mathrm{~h}$, the volume of mitochondria decreased and the number of mitochondria increased, indicating mitochondrial fragmentation. SIRT1 overexpression treatment attenuated PA-induced mitochondrial fragmentation. After si-Opa1 transfection, mitochondrial fragmentation increased (Fig. 5A).

\section{BBR improved hepatic IR through the SIRT1/Opa1 pathway}

To explore whether BBR can improve hepatic IR by regulating the SIRT1/Opa1 pathway, BBR treatment $(10 \mu \mathrm{M})$ was given for $24 \mathrm{~h}$ (Fig. 6A) based on the PA model, followed by SIRT1 silencing intervention. The results revealed that when BBR treatment was given based on PA stimulation, SIRT1 and Opa1 protein expression (Fig. 6B and 6C), ATP content (Fig. 6F), and MMP (Fig. 6G) were all increased, and pAKT sensitivity to insulin (Fig. 6D) and glucose consumption (Fig. 6E) were increased significantly. However, all these effects were reduced after SIRT1 silencing. These results suggested that BBR improves hepatic IR by regulating the SIRT1/Opa1 pathway.

BBR improved hepatic IR and increased SIRT1 and Opa1 expression in the liver of the $\mathrm{db} / \mathrm{db}$ mice 
Our data revealed that the body weight, FBG, LDL, T-CHO, and TG in the $\mathrm{db} / \mathrm{db}$ mice were significantly increased compared with those in the control group (Fig. 7A-E). Moreover, the $\mathrm{db} / \mathrm{db}$ mice exhibited impaired glucose and insulin tolerance (Fig. 7F and 7G). An increased expression of PEPCK and G6pase was also observed (Fig. 7H and Fig. 7I). However, compared with the $\mathrm{db} / \mathrm{db}$ group, 4-week-old BBR-treated $\mathrm{db} / \mathrm{db}$ mice exhibited significant hypoglycaemic and lipid-lowering effects and an improvement in results of OGTT and ITT.

The results of HE staining of the liver revealed that the cells in the $\mathrm{db} / \mathrm{db}$ mice liver were highly swollen and the cellular matrix was highly porous and showed vacuole-like changes. Hepatocytes had fatty vacuoles of various sizes and clear boundaries and the hepatocyte cords were disorderly arranged, which mainly manifested as cell oedema and hepatic steatosis. After treatment with BBR, the liver cells appeared neatly arranged and uniformly sized, the fatification significantly improved, and the degree of liver tissue damage was greatly improved (Fig. 8A). Liver cell electron microscopy showed that the mitochondrial ridges in the control mice liver cells were dense and varied in size. The volume of mitochondria in the $\mathrm{db} / \mathrm{db}$ group became significantly smaller and the ridges became sparse. Some mitochondria were broken and fused, and the condition of sparse mitochondrial cristae was significantly improved after BBR treatment (Fig. 8B).

The expression of Opa1 was decreased, whereas that of Drp1 was increased in the $\mathrm{db} / \mathrm{db}$ mice, suggesting a disordered mitochondrial dynamics. The expression of SIRT1 decreased in the $\mathrm{db} / \mathrm{db}$ mice. After treatment with BBR, the expression of 
SIRT1/Opa1 increased, whereas that of Drp1 decreased (Fig. 8C). These results suggested that the SIRT1/Opa1 pathway is related to hepatic IR.

\section{Discussion}

Excess serum free fatty acids (FFA) play a key role in obesity and type 2 diabetes [18]. An excess of FFAs in blood causes increased accumulation of lipid metabolites in the liver and skeletal muscles and can further worsen IR, which is the core defect in type 2 diabetes mellitus. Palmitate (PAs), a representative of saturated fatty acids, is used for diabetic state simulation [19]. HepG2 cells are human-derived hepatic embryonic tumour cells with a phenotype similar to that of hepatocytes. In addition, the HepG2 cells are not interfered by other factors such as ageing and are used for the study of hepatic IR. In this study, we demonstrated that the protein expression of SIRT1/Opal decreases in the liver of HFD-fed mice, $\mathrm{db} / \mathrm{db}$ mice, and PA-induced HepG2 cells. Opa1 deficiency in HepG2 cells induces hepatocyte IR and mitochondrial dysfunction. SIRT1 overexpression and BBR treatment can activate Opal and improve hepatocyte IR and mitochondrial functions. Opal and SIRT1 silencing could partly reverse these effects of SIRT1 and BBR, respectively. Overall, BBR improves hepatic IR through the SIRT1/Opal pathway in hepatic insulin-resistant mice and PA-induced HepG2 cells. Based on these data, we propose that Opa1 is a potential target in diabetes drug development.

IR is a condition in which the sensitivity of peripheral tissues (liver, muscle, and adipose tissues) to insulin decreases[20]. Given the key role of the liver in glucose 
production and lipid metabolism, hepatic IR is considered as the factor involved in the development of IR and diabetes[21]. Therefore, a better understanding of the mechanism of hepatic IR may help in elucidating a new therapeutic target for the treatment and prevention of diabetes. Insulin binds to the insulin receptor and promotes the autophosphorylation of IR tyrosine residues in the hepatocytes[22,23]. Then, tyrosine kinase phosphorylates insulin receptor substrate 2 (IRS-2), which can further activate phosphatidylinositol 3-kinase (PI3K) and AKT/protein kinase $\mathrm{B}$ and increase glucose transporter 2 (GLUT2) membrane transport. The insulin signalling pathway promotes blood glucose entry into the hepatocytes and regulates the normal metabolism of lipid and glucose[24,25]. Excess lipid accumulation in the liver may impair the hepatic insulin signal pathway and cause hepatic IR [26]. In our study, the HFD-fed mice and $\mathrm{db} / \mathrm{db}$ mice exhibited increased FBG, impaired glucose tolerance, hyperinsulinemia, and high gluconeogenic enzyme expression (PEPCK and G6pase). Additionally, PA-treated HepG2 cells demonstrated AKT insensitivity in response to insulin and decreased glucose consumption, indicating that the IR cell model can be successfully constructed using HepG2 cells. Mitochondrial dynamics imbalance is an important initiating cause of peripheral IR and type 2 diabetes [27-29]. Our results also validate the abnormality of mitochondrial fusion and fission during hepatic IR. BBR and SIRT1 play an important role in the preservation of mitochondrial dynamics and improvement of hepatic IR.

Opal is a GTPase anchored to the IMM, which has long and short isoforms. In addition to being responsible for the IMM fusion, Opal is involved in maintaining the 
crista structure and protecting cells from apoptosis [30,31]. Opa1 deficiency is closely related to the occurrence and development of diabetes. Zhang et al[32] reported that the loss of Opal in pancreatic $\beta$ cells impairs glucose-stimulated ATP production and insulin secretion, which then develop into hyperglycaemia. Ding et $\mathrm{al}[33]$ reported that the Opal expression was reduced in the heart of diabetic rats, and after treatment with mitochondrial fusion activator M1, the Opal expression was increased and the mitochondrial function and diabetic cardiomyopathy were improved; however, all these effects were weakened after Opal silencing. In our study, the downregulation of Opal was observed in the HFD-fed mice and $\mathrm{db} / \mathrm{db}$ mice. Furthermore, we observed an increased expression of gluconeogenic genes, such as PEPCK and G6pase, in Opa1-silenced HepG2 cells. The sensitivity of AKT to insulin also reduced in the Opa1-silenced HepG2 cells. Therefore, we propose that Opa1 deficiency is associated with a pattern of hepatic IR in animal and cell models. Under these conditions, we also observed that Opa1 deficiency changes mitochondrial fusion and fission. Further studies are required to explore whether Opal deficiency reduces insulin signalling in liver tissues and induces susceptibility to IR in Opal liver-KO mice.

The human sirtuin isoform SIRT1-7 has been related to type 2 diabetes[34]. SIRT2 is mainly distributed in the cytoplasm; SIRT1, SIRT6, and SIRT7 are mainly located in the nucleus; and SIRT3-5 are located in the mitochondria. Previous studies have shown that SIRT3 regulates mitochondrial dynamics by deacetylating and activating Opal during stress[35], whereas SIRT4 regulates mitochondrial quality 
control and mitophagy by interacting with Opa1 [36]. SIRT5 overexpression prevents mitochondrial fragmentation and protects against mitophagy by increasing Mfn2 and Opa1, whereas these effects were reversed after SIRT5 silencing [37]. However, evidence to confirm that SIRT1 regulates Opa1 is lacking. Another major finding of our study is that SIRT1 activates Opa1, improves hepatic IR, inhibits PA-induced mitochondrial fragmentation, and alleviates mitochondrial dysfunction in PA-induced HepG2 cells. These effects were partly reversed when Opa1 was silenced. Thus, the activation of Opa1 by SIRT1 plays a key role in alleviating hepatocyte IR.

Berberine (BBR) has received increasing attention for its potential to treat liver steatosis, dyslipidaemia, and diabetes. BBR and its derivatives have been shown to reduce hepatic steatosis in HepG2 cells[38], HFD-fed rats[39-41], and patients with nonalcoholic fatty liver[42], and SIRT1 is the key regulator in liver lipid metabolism[43]. Therefore, to investigate whether BBR can reduce blood glucose and improve hepatic IR through SIRT1 and whether SIRT1 is related to mitochondrial dynamics-related proteins, we used the SIRT1 silencing technology to observe the relationship of SIRT1 with Opa1 in PA-induced HepG2 cells. The results revealed that BBR increased the SIRT1 and Opa1 protein expression, ATP content, and MMP. The sensitivity of pAKT to insulin and glucose consumption significantly increased. However, these effects were reversed after SIRT1 silencing, suggesting that BBR improves hepatic IR by upregulating the SIRT1/Opa1 pathway.

To validate the effect in vivo, we treated the $\mathrm{db} / \mathrm{db}$ mice with BBR. The results revealed that BBR treatment significantly improves the blood glucose and liver IR of 
mice. The expression of Opal and SIRT1 was upregulated after BBR treatment, whereas the expression of Drp1 was downregulated. The mitochondrial fragmentation was significantly improved after BBR treatment. BBR improves the development of hepatic IR, which may be related to the balance of mitochondrial dynamics and upregulation of the SIRT1/Opa1 signalling pathway. Overall, these results suggest that BBR can improve hepatic IR by regulating the SIRT1/Opa1 pathway in $\mathrm{db} / \mathrm{db}$ mice and PA-induced HepG2 cells.

\section{Conclusion}

In summary, our results showed that Opal silencing-mediated mitochondrial fusion/fission imbalance could lead to hepatocyte IR, whereas SIRT1 improves hepatocyte IR by activating Opa1. As an effective hypoglycaemic drug, BBR may improve hepatic IR by regulating the SIRT1/Opal pathway and thus can be used to treat type 2 diabetes.

\section{Availability of data and materials}

The datasets used and/or analysed during the current study are available from the corresponding author on reasonable request.

\section{Acknowledgments}

The authors thank Prof. Li Chen for providing laboratory equipment and technical assistance. 


\section{Funding}

This work was supported by the Science and technology development projects of Jilin Province (20200201483JC, 20200404097YY, 20200201456JC, 2019SCZT042).

\section{Author information}

\section{Affiliations}

Department of Pharmacology, School of Basic Medical Sciences, Jilin University, Changchun, China

Jia Xu, Zhiyi Yu, Yueqi Guan, Yuqian Lv, Meishuang Zhang, Ming Zhang, Li Chen, Fengying Guan

Department of Clinical Laboratory, the Second Clinical Hospital Affiliated to Jilin University, Changchun, China

Xiaoyan lv

Department of Pediatric Endocrinology, The First Clinical Hospital Affiliated to Jilin University, Changchun, China

Yining Zhang

\section{Contributions}

Xiaoyan Lv, Yining Zhang and Fengying Guan designed the experimens; Ming Zhang and Li Chen cotributed this article as expert consultation. Jia Xu, Yining Zhang, Zhiyi Yu, Yueqi Guan, Yuqian Lv and Meishuang Zhang contributed to the data collection; Jia $\mathrm{Xu}$ and Xiaoyan lv performed the data analysis and interpreted the results; Jia Xu and Fengying Guan wrote the manuscript. All authors read and approved the final 
manuscript.

\section{Corresponding author}

Correspondence to Fengying Guan or Xiaoyan lv.

\section{Ethics declarations}

\section{Ethics approval and consent to participate}

All animal studies were conducted following the Guide for the Care and Use of Laboratory Animals and were approved by the Institutional Animal Care and Use Committee of Jilin University (permit number, SYSK 2013-0005).

\section{Consent for publication}

Not applicable.

\section{Competing interests}

The authors declare that they have no competing interests.

\section{Figure legends}

\section{Figure 1. A HFD-induced hepatic insulin resistance mice model was established}

(A) Starting from week 5, the weight of the HFD-fed mice increased significantly. Data are expressed as means \pm SEM $(n=10)$; compared with the LFD group, ${ }^{* * P}<$ $0.01,{ }^{* * *} \mathrm{P}<0.001$. (B) For HFD-obese mice, a significant increase in fasting blood glucose was observed at week 10. Data are expressed as means \pm SEM $(n=6)$; compared with the LFD group, $* * \mathrm{P}<0.01$. (C, D) HFD-fed mice showed impaired glucose tolerance and hyperinsulinemia. Data are expressed as means $\pm \operatorname{SEM}(n=6)$; 
compared with the LFD 0 min group, ${ }^{*} \mathrm{P}<0.05$; compared with the LFD 30 min group, ${ }^{\#} \mathrm{P}<0.05$. (E) qPCR was utilised showing a sharp increase in PEPCK and G6pase in HFD-fed mice. Data are expressed as means \pm SEM $(n=6)$. Compared with the LFD group, ${ }^{*} \mathrm{P}<0.05, * * * \mathrm{P}<0.001$. (F) HE staining results of LFD- and HFD-fed mice (200x).


LFD

HFD

Figure 2. Downregulation of SIRT1 and Opa1 was related to the imbalance of mitochondrial dynamics and hepatic insulin resistance

(A) WB grayscale picture of SIRT1, Opa1, Mfn1, and Drp1. (B, C, D) The expression of SIRT1, Opa1, and Mfn1 in HFD-fed mice decreased. Data are expressed as means \pm SEM $(\mathrm{n}=3)$; compared with the LFD group, ${ }^{*} \mathrm{P}<0.05,{ }^{* *} \mathrm{P}<$ 0.01. (E) The expression of Drp1 in HFD-fed mice increased. Data are expressed as means $\pm \operatorname{SEM}(\mathrm{n}=3)$; compared with the LFD group, $* \mathrm{P}<0.05$. 

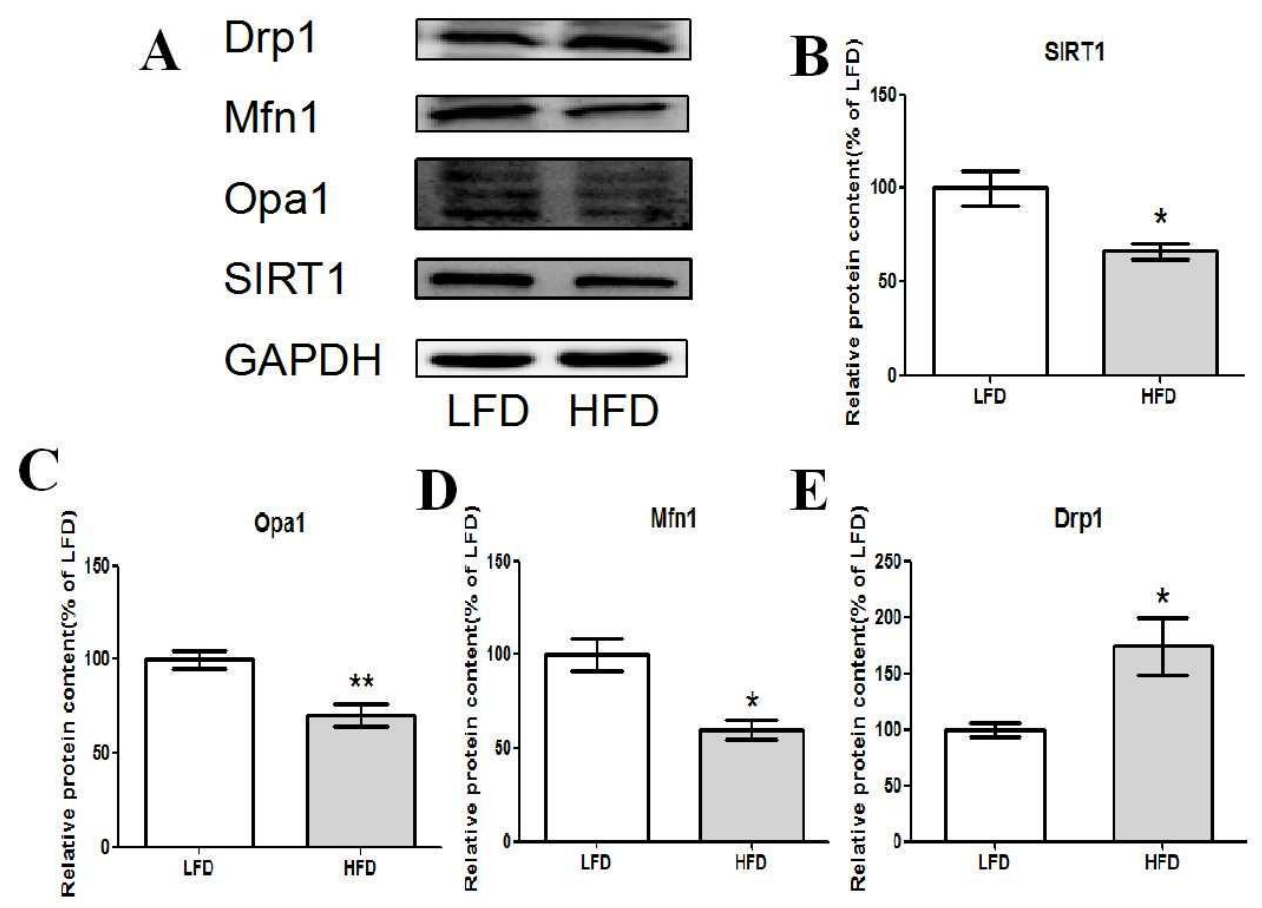

Figure 3. Opa1 deficiency changed mitochondrial fusion/fission and impaired insulin signalling in the HepG2 cells

(A) Cells were transfected with Opal-siRNA for $48 \mathrm{~h}$, and the transfection efficiency was approximately 55\%. The protein expression of NDUFA9 decreased and that of Drp1 increased in the si-Opal group. $(\mathrm{B}, \mathrm{C})$ ATP content and mitochondrial membrane potential $(200 \times)$ decreased in the si-Opal group. (D) An increase in the mRNA expression of PEPCK and G6pase was observed in the si-Opal group. (E) A marked reduction in phosphorylation of AKT in response to insulin was observed in the si-Opal group. Data are expressed as mean \pm SD of 3 independent experiments with 3 determinations for each experiment; compared with the si-Opal group, ${ }^{*} \mathrm{P}<$ $0.05, * * \mathrm{P}<0.01, * * * \mathrm{P}<0.001$ 

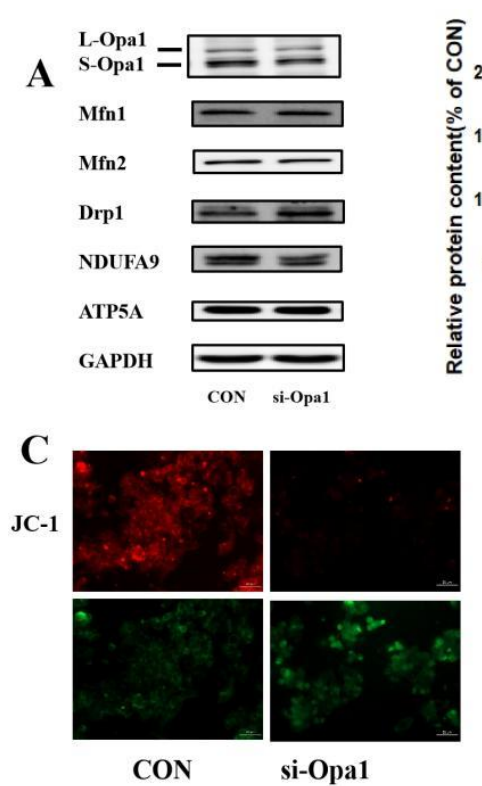

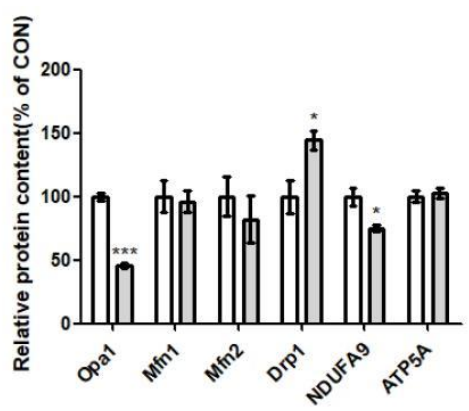

D

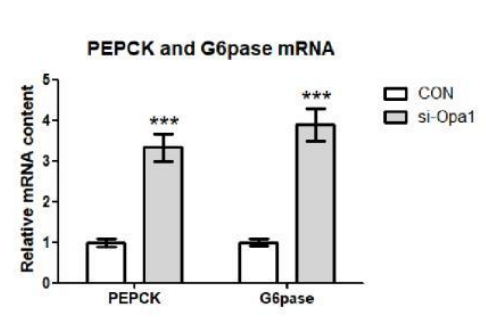

B

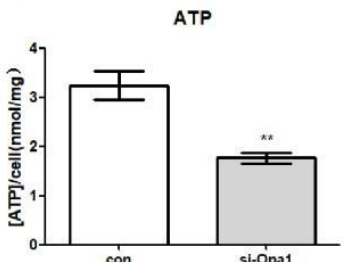

$\mathbf{E}$

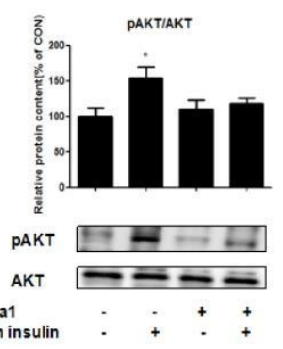

Figure 4. Overexpression of SIRT1 activated Opa1 and ameliorated PA-induced insulin resistance in the HepG2 cells

(A) The influence of PA-induced hepatic insulin resistance was assessed using the MTT assay. Data are expressed as mean \pm SD of 3 independent experiments with 6 determinations for each experiment; compared with the control group, ${ }^{*} \mathrm{P}<0.05$, ***P $<0.001$. (B) After treatment of HepG2 cells with $0.3 \mathrm{mM}$ PA, a markedly reduced phosphorylation of AKT in response to insulin appeared, which indicated that the IR model was established. Data are expressed as mean \pm SD of 3 independent experiments with 3 determinations for each experiment; compared with the control group, ${ }^{*} \mathrm{P}<0.05$. $(\mathrm{C}, \mathrm{D})$ The protein was extracted from cells and SIRT1 and Opa1 were measured and analysed using western blot. Data are expressed as mean \pm SD of 3 independent experiments with 3 determinations for each experiment; compared with the control group, ${ }^{*} \mathrm{P}<0.05,{ }^{* *} \mathrm{P}<0.01$; compared with the $\mathrm{PA}$ group, ${ }^{\#} \mathrm{P}<0.05,{ }^{\# \#} \mathrm{P}$ $<0.001$. (E) Results of phosphorylation of AKT in response to insulin in each group. 
Data are expressed as mean \pm SD of 3 independent experiments with 3 determinations for each experiment. (F) The glucose consumption results in each group. Data are expressed as mean $\pm \mathrm{SD}$ of 3 independent experiments with 4 determinations for each experiment; compared with the control group, $* * * \mathrm{P}<0.001$; compared with the PA group, ${ }^{\# \#} \mathrm{P}<0.001$; compared with the $\mathrm{PA}+$ oe-SIRT1 group, ${ }^{\& \& \&} \mathrm{P}<0.001$. (G) ATP content in each group. Data are expressed as mean \pm SD of 3 independent experiments with 3 determinations for each experiment; compared with the control group, ${ }^{* * *} \mathrm{P}<0.001$; compared with the PA group, ${ }^{\# \# \# P}<0.001$; compared with the $\mathrm{PA}+$ oe-SIRT1 group, ${ }^{\& \& \&} \mathrm{P}<0.001$.
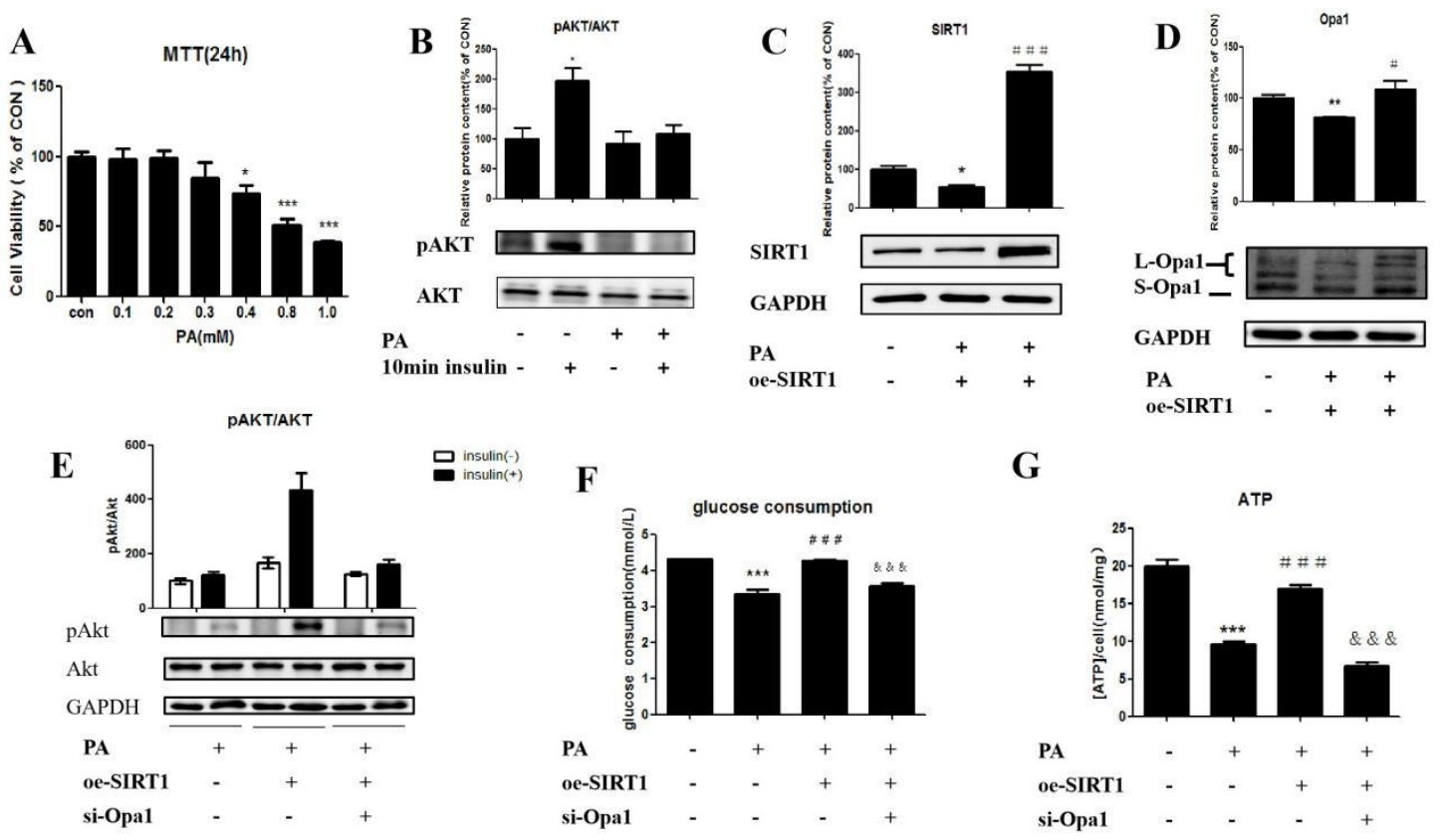

G

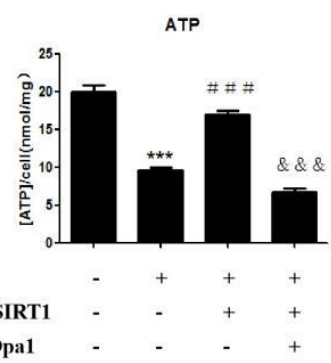

Figure 5. Overexpression of SIRT1 improved PA-induced mitochondrial morphology and mitochondrial membrane potential in the HepG2 cells

(A) Mitochondrial morphology in each group, transfected with mitochondrially targeted Mito-DsRed fluorescent protein (bar $=10 \mu \mathrm{m})$. (B) Mitochondrial membrane 
potential detected by JC-1 in each group $(200 \times)$.

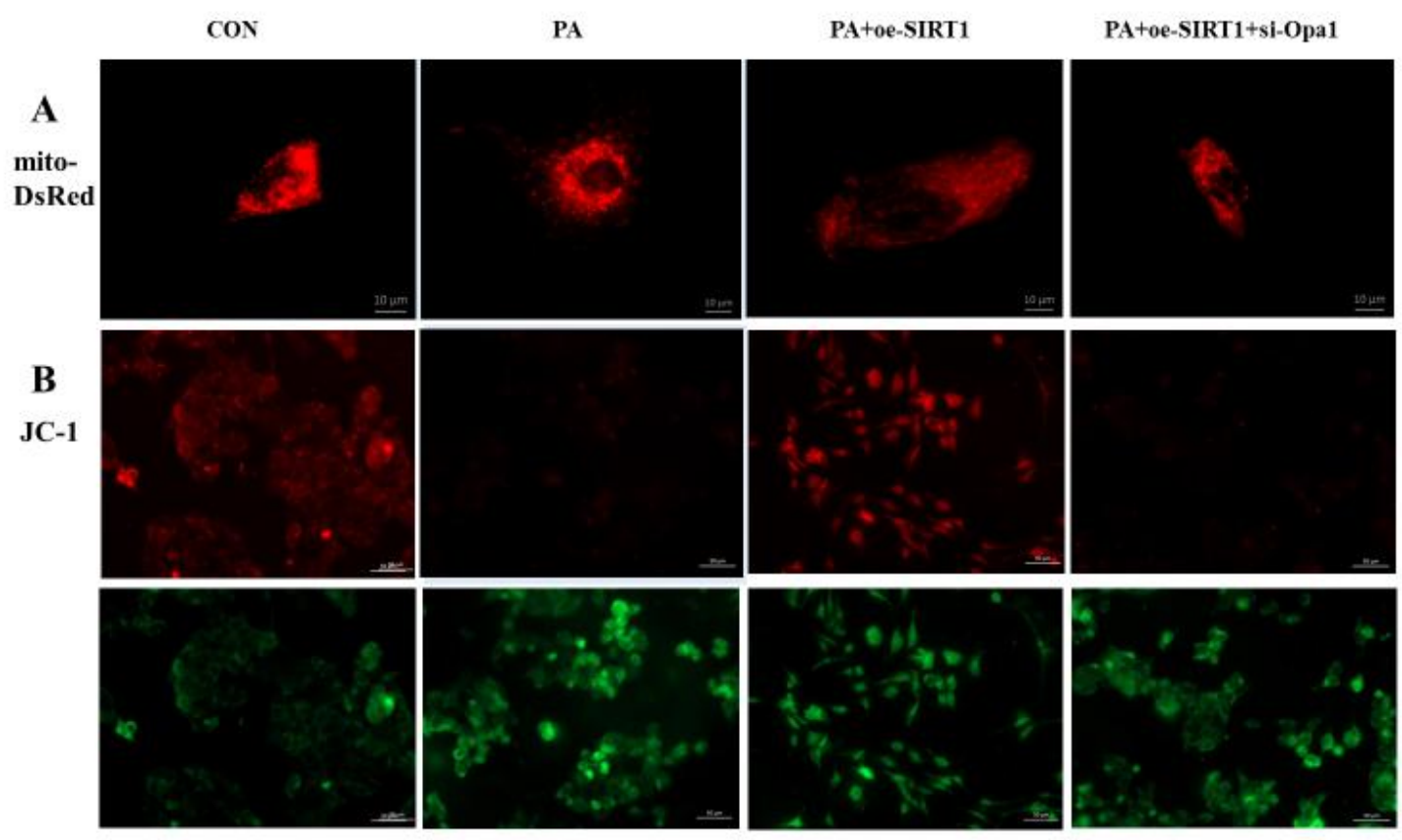

Figure 6. BBR improved hepatic insulin resistance through the SIRT1/Opa1 pathway

(A) The influence of BBR on HepG2 cells was assessed using the MTT assay. Data are expressed as mean \pm SD of 3 independent experiments with 6 determinations for each experiment; compared with the control group, $* \mathrm{P}<0.05$. (B,C) The protein was extracted from the cells, and SIRT1 and Opa1 were measured and analysed using western blot. Data are expressed as mean $\pm \mathrm{SD}$ of 3 independent experiments with 3 determinations for each experiment; compared with the control group, $\quad * * \mathrm{P}<0.01$; compared with the PA group, ${ }^{\# \#} \mathrm{P}<0.01,{ }^{\# \#} \mathrm{P}<0.001$; compared with the PA + BBR group, ${ }^{\& \&} \mathrm{P}<0.01, \quad{ }^{\& \&} \mathrm{P}<0.001$. (D) Results of AKT phosphorylation in response to insulin in each group. Data are expressed as mean \pm SD of 3 independent experiments with 3 determinations for each experiment. (E) The glucose consumption results in 
each group. Data are expressed as mean \pm SD of 3 independent experiments with 4 determinations for each experiment; compared with the control group, ${ }^{* * *} \mathrm{P}<0.001$; compared with the PA group, ${ }^{\# \#} \mathrm{P}<0.001$; compared with the PA + BBR group, ${ }^{\& \& \&} \mathrm{P}$ $<0.001$. (F) ATP content in each group. Data are expressed as mean $\pm \mathrm{SD}$ of 3 independent experiments with 3 determinations for each experiment; compared with the control group, ${ }^{* * *} \mathrm{P}<0.001$; compared with the PA group, ${ }^{\# \#} \mathrm{P}<0.001$; compared with the PA + BBR group, ${ }^{\& \& \&} \mathrm{P}<0.001$. (G) Mitochondrial membrane potential detected by JC-1 in each group (200×).

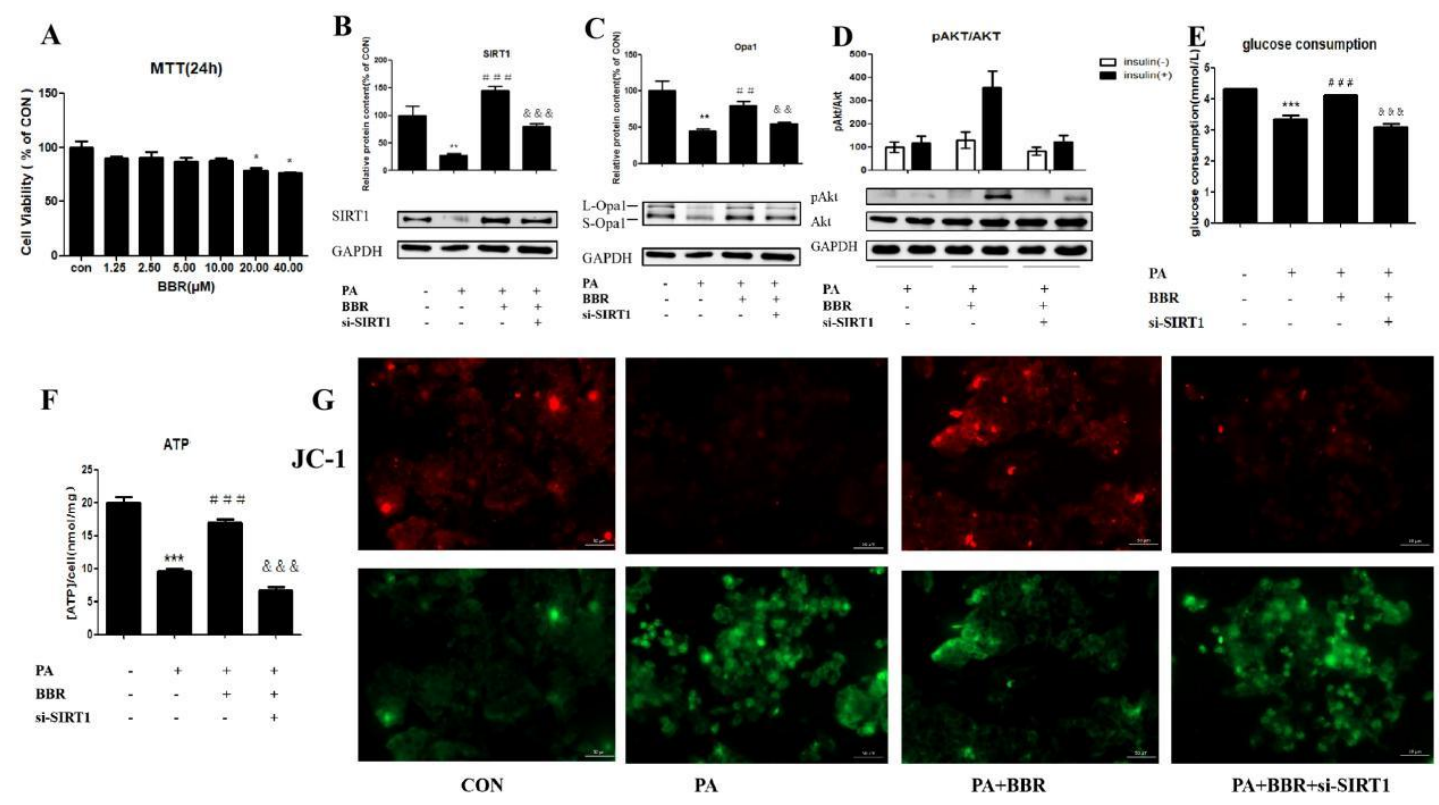

Figure 7. Berberine improved hepatic insulin resistance in the $\mathrm{db} / \mathrm{db}$ diabetic mice

(A) Body weight was tested for 4 weeks. (B-E) FBG, LDL, T-CHO, and TG were observed at the end of week 12. (F, G) Plasma glucose concentrations in different phases were measured in the oral glucose tolerance test (OGTT) and insulin tolerance test (ITT) at week 12. (H, I) qPCR was utilised showing the mRNA expression of 
PEPCK and G6pase. Data are expressed as means \pm SEM $(n=6)$; compared with the CON group, ${ }^{*} \mathrm{P}<0.05,{ }^{* *} \mathrm{P}<0.01,{ }^{* * *} \mathrm{P}<0.001$; compared with the $\mathrm{db} / \mathrm{db}$ group, ${ }^{\#} \mathrm{P}$ $<0.05,{ }^{\#} \mathrm{P}<0.01$
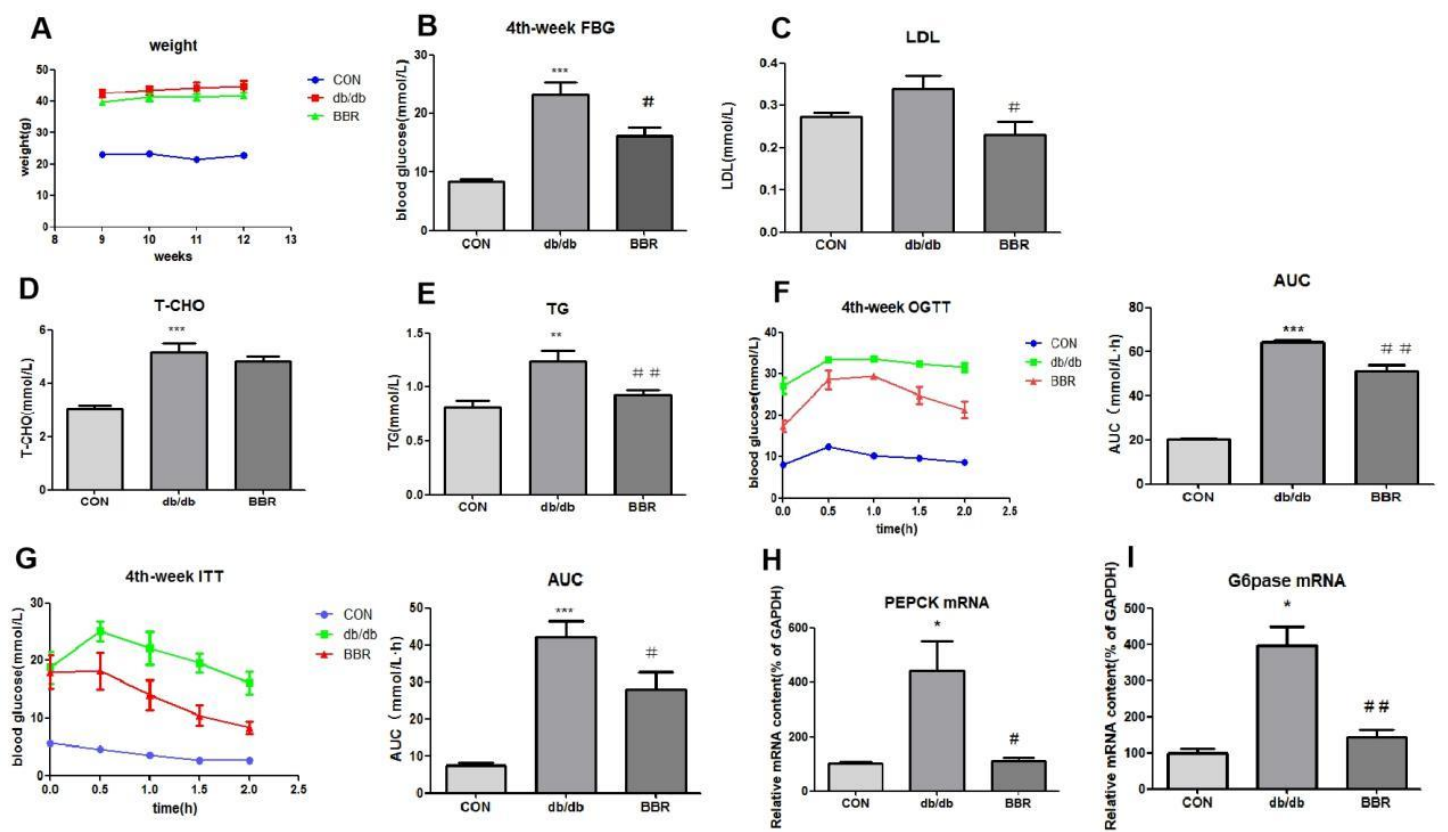

Figure 8. Downregulation of SIRT1 and Opa1 in the $\mathrm{db} / \mathrm{db}$ mice

(A) HE staining of the mice $(200 \times)$. (B) Injury of mitochondria is shown in electron microscopic images and marked with arrows. (C) The expression of SIRT1, Opa1, and Drp1 in the liver of the mice. Data are expressed as means \pm SEM $(n=3)$; compared with the CON group, ${ }^{*} \mathrm{P}<0.05$, **P $<0.01$; compared with the $\mathrm{db} / \mathrm{db}$ group, ${ }^{\#} \mathrm{P}<0.05$. 

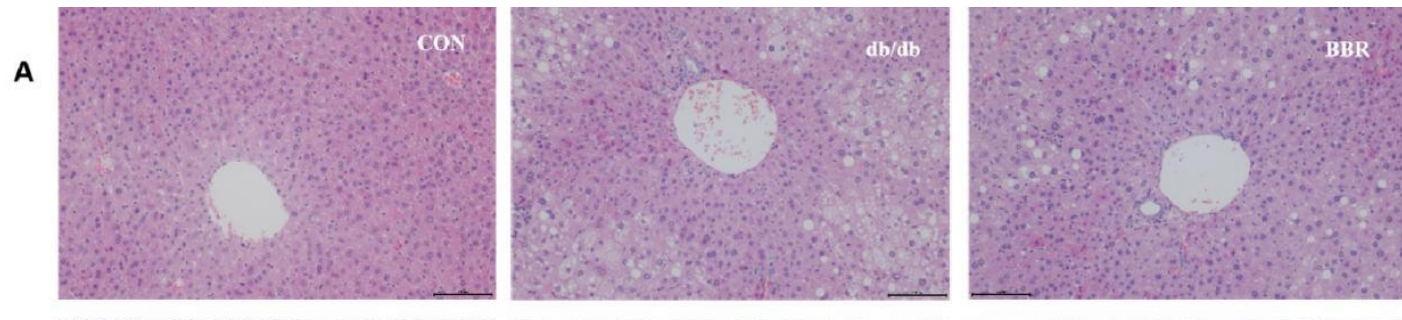

B
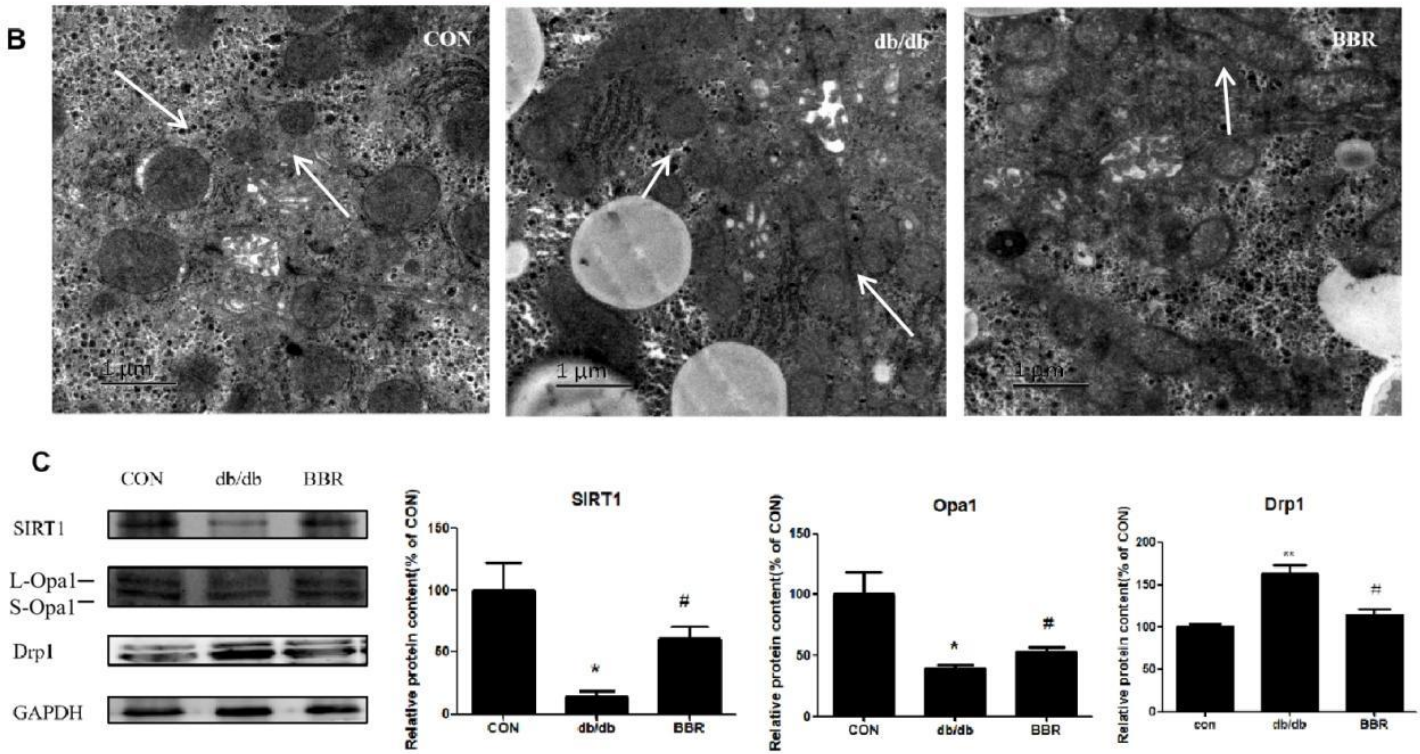

\section{Reference}

[1] Srinivasan S, Guha M, Kashina A, Avadhani NG. Mitochondrial dysfunction and mitochondrial dynamics-The cancer connection. Biochim Biophys Acta Bioenerg. 2017;1858:602-614.

[2] Liesa M, Shirihai OS. Mitochondrial dynamics in the regulation of nutrient utilization and energy expenditure. Cell Metab. 2013;17:491-506.Crescenzo R,

[3] Bianco F, Mazzoli A, Giacco A, Liverini G, Iossa S. A possible link between hepatic mitochondrial dysfunction and diet-induced insulin resistance. Eur J Nutr. 2016;55:1-6.

[4] Rovira-Llopis S, Bañuls C, Diaz-Morales N, Hernandez-Mijares A, Rocha M, Victor VM. Mitochondrial dynamics in type 2 diabetes: Pathophysiological 
implications. Redox Biol. 2017;11:637-645.

[5] Kulkarni SS, Joffraud M, Boutant M, Ratajczak J, Gao AW, Maclachlan C, et al. Mfn1 Deficiency in the Liver Protects Against Diet-Induced Insulin Resistance and Enhances the Hypoglycemic Effect of Metformin. Diabetes. 2016;65:3552-3560.

[6] Sebastián D, Hernández-Alvarez MI, Segalés J, Sorianello E, Muñoz JP, Sala D, et al. Mitofusin 2 (Mfn2) links mitochondrial and endoplasmic reticulum function with insulin signaling and is essential for normal glucose homeostasis. Proc Natl Acad Sci U S A. 2012;109:5523-5528.

[7] Wang L, Ishihara T, Ibayashi Y, Tatsushima K, Setoyama D, Hanada Y, et al. Disruption of mitochondrial fission in the liver protects mice from diet-induced obesity and metabolic deterioration. Diabetologia. 2015b;58:2371-2380.

[8] Yuan Y, Cruzat VF, Newsholme P, Cheng J, Chen Y, Lu Y. Regulation of SIRT1 in aging: Roles in mitochondrial function and biogenesis. Mech Ageing Dev. 2016;155:10-21.

[9] Sui M, Chen G, Mao X, Wei X, Chen Y, Liu C, et al. Gegen Qinlian Decoction Ameliorates Hepatic Insulin Resistance by Silent Information Regulator1 (SIRT1)-Dependent Deacetylation of Forkhead Box O1 (FOXO1). Med Sci Monit. 2019;25:8544-8553.

[10] Jung TW, Ahn SH, Shin JW, Kim HC, Park ES, Abd El-Aty AM, et al. Protectin DX ameliorates palmitate-induced hepatic insulin resistance through AMPK/SIRT1-mediated modulation of fetuin-A and SeP expression. Clin Exp 
Pharmacol Physiol. 2019;46:898-909.

[11] Kitada M, Koya D. SIRT1 in Type 2 Diabetes: Mechanisms and Therapeutic Potential. Diabetes Metab J. 2013;37:315-325.

[12] Ding M, Feng N, Tang D, Feng J, Li Z, Jia M, et al. Melatonin prevents Drp1-mediated mitochondrial fission in diabetic hearts through SIRT1-PGC1 $\alpha$ pathway. J Pineal Res. 2018;65:e12491.

[13] Tu J, Zhu S L, Zhou X M. Differentiated hypoglycemic effects of baicalin, berberine and puerarin on insulin-resistance HepG2 cells. China J Chi Mat Med. 2018;43:4097-4103.

[14] Qin X, Zhao Y, Gong J, et al. Berberine Protects Glomerular Podocytes via Inhibiting Drp1-Mediated Mitochondrial Fission and Dysfunction. Theranostics. 2019;9:1698-1713.

[15] Qin X, Jiang M, Zhao Y, et al. Berberine protects against diabetic kidney disease via promoting PGC-1 $\alpha$-regulated mitochondrial energy homeostasis. $\mathrm{Br} \mathrm{J}$ Pharmacol. 2019;16:21-23.

[16] Si M, Yan Y, Tang L, Wu H, Yang B, He Q. A novel indole derivative compound GY3 improves glucose and lipid metabolism via activation of AMP-activated protein kinase pathway. Eur J Pharmacol. 2013;698:480-488.

[17] Petersen MC, Shulman GI. Mechanisms of Insulin Action and Insulin Resistance. Physiol Rev. 2018;98:2133-223.

[18] Daniele G, Eldor R, Merovci A, Clarke GD, Xiong J, Tripathy D, et al. Chronic reduction of plasma free fatty acid improves mitochondrial function and 
whole-body insulin sensitivity in obese and type 2 diabetic individuals. Diabetes. $2014 ; 63: 2812-2820$

[19] Yang Z, Chen X, Chen Y, Zhao Q. Decreased irisin secretion contributes to muscle insulin resistance in high-fat diet mice. Int J Clin Exp Pathol. 2015; $8: 6490-6497$.

[20] Barseem NF, Helwa MA. Homeostatic model assessment of insulin resistance as a predictor of metabolic syndrome: Consequences of obesity in children and adolescents[J]. Egyptian Pediatric Association Gazette, 2015;63:19-24.

[21] Ibarra-Reynoso LeR, Pisarchyk L, Pérez-Luque EL, Garay-Sevilla ME, Malacara JM. Whole-body and hepatic insulin resistance in obese children. PLoS One. 2014;9:e113576.

[22] Morakinyo A O, Samuel T A, Adekunbi D A . Magnesium upregulates insulin receptor and glucose transporter-4 in streptozotocin-nicotinamide-induced type-2 diabetic rats[J]. Nephron Clinical Practice.2018;52:6-16.

[23] Wang J, Zou T, Yang HX, Gong YZ, Xie XJ, Liu HY, et al. Insulin receptor binding motif tagged with IgG4 Fc (Yiminsu) works as an insulin sensitizer to activate AKT signaling in hepatocytes. Genet Mol Res. 2015a;14:8819-8828.

[24] Cai S, Sun W, Fan Y, Guo X, Xu G, Xu T, et al. Effect of mulberry leaf (Folium Mori) on insulin resistance via IRS-1/PI3K/Glut-4 signalling pathway in type 2 diabetes mellitus rats. Pharm Biol. 2016;54:2685-2691.

[25] Chen L, Zheng S, Huang M , et al. $\beta$-ecdysterone from, Cyanotis arachnoidea, exerts hypoglycemic effects through activating IRS-1/AKT/GLUT4 and 
IRS-1/AKT/GLUT2 signal pathways in KK-Ay mice[J]. Journal of Functional Foods, 2017, 39:123-132.

[26] Chen L, Chen XW, Huang X, Song BL, Wang Y. Regulation of glucose and lipid metabolism in health and disease. Sci China Life Sci. 2019;62:1420-1458.

[27] Zorzano A, Liesa M, Palacín M. Mitochondrial dynamics as a bridge between mitochondrial dysfunction and insulin resistance. Arch Physiol Biochem. 2009;115:1-12.

[28] Roy M, Reddy PH, Iijima M, Sesaki H. Mitochondrial division and fusion in metabolism. Curr Opin Cell Biol. 2015;33:111-118.

[29] Wang $\mathrm{CH}$, Wang CC, Wei YH. Mitochondrial dysfunction in insulin insensitivity: implication of mitochondrial role in type 2 diabetes. Ann N Y Acad Sci. 2010;1201:157-165.

[30] Frezza C, Cipolat S, Martins de Brito O, Micaroni M, Beznoussenko GV, Rudka $\mathrm{T}$, et al. OPA1 controls apoptotic cristae remodeling independently from mitochondrial fusion. Cell. 2006;126:177-189.

[31] Olichon A, Baricault L, Gas N, Guillou E, Valette A, Belenguer P, et al. Loss of OPA1 perturbates the mitochondrial inner membrane structure and integrity, leading to cytochrome $\mathrm{c}$ release and apoptosis. $\mathrm{J}$ Biol Chem. $2003 ; 278: 7743-7746$.

[32] Zhang Z, Wakabayashi N, Wakabayashi J, et al. The dynamin-related GTPase Opa1 is required for glucose-stimulated ATP production in pancreatic beta cells. Mol Biol Cell. 2011;22:2235-2245. 
[33] Ding M, Liu C, Shi R, et al. Mitochondrial fusion promoter restores mitochondrial dynamics balance and ameliorates diabetic cardiomyopathy in an optic atrophy 1-dpendent way. Acta Physiol (Oxf). 2019;12:e13428.

[34] Farghali H, Kemelo MK, Canová NK. SIRT1 Modulators in Experimentally Induced Liver Injury. Oxid Med Cell Longev. 2019;2019:8765954.

[35] Samant SA, Zhang HJ, Hong Z, Pillai VB, Sundaresan NR, Wolfgeher D, et al. SIRT3 deacetylates and activates OPA1 to regulate mitochondrial dynamics during stress. Mol Cell Biol. 2014;34:807-819.

[36] Lang A, Anand R, Altinoluk-Hambüchen S, Ezzahoini H, Stefanski A, Iram A, et al. SIRT4 interacts with OPA1 and regulates mitochondrial quality control and mitophagy. Aging (Albany NY). 2017;9:2163-2189.

[37] Polletta L, Vernucci E, Carnevale I, Arcangeli T, Rotili D, Palmerio S, et al. SIRT5 regulation of ammonia-induced autophagy and mitophagy. Autophagy. 2015;11:253-270.

[38] Brusq J M, Ancellin N, Grondin P, et al. Inhibition of lipid synthesis through activation of AMP kinase: an additional mechanism for the hypolipidemic effects of berberine. J Lipid Res. 2006;47:1281-1288.

[39] Chang X, Yan H, Fei J, et al. Berberine reduces methylation of the MTTP promoter and alleviates fatty liver induced by a high-fat diet in rats. J Lipid Res. 2010;51:2504-2515.

[40] Xia X, Yan J, Shen Y, et al. Berberine improves glucose metabolism in diabetic rats by inhibition of hepatic gluconeogenesis. PLoS ONE. 2011;6:16556. 
[41] Yuan X, Wang J, Tang X, et al. Berberine ameliorates nonalcoholic fatty liver disease by a global modulation of hepatic mRNA and lncRNA expression profiles. J Transl Med. 2015;13:24.

[42] Yan H M, Xia M F, Wang Y, et al. Efficacy of berberine in patients with non-alcoholic fatty liver disease. PLoS ONE. 2015;10:134172.

[43] Sun Y, Xia M, Yan H, et al. Berberine attenuates hepatic steatosis and enhances energy expenditure in mice by inducing autophagy and fibroblast growth factor 21. Br J Pharmacol. 2018;175:374-387. 